\title{
AN INVESTIGATION OF DIVERSITY AND BIOECOLOGY OF ARANEOFAUNA OF PATHIRAMANAL ISLAND IN VEMBANAD LAKE, A RAMSAR SITE, KERALA, INDIA
}

\author{
Jobi J. Malamel ${ }^{\mathrm{a} *}$ and Ambalaparambil Vasu Sudhikumar ${ }^{\mathrm{b}}$
}

${ }^{\mathrm{a} D i v i s i o n}$ of Arachnology, Department of Zoology, Sacred Heart College, Thevara, Cochin, Kerala 682 013, India; ${ }^{\mathrm{b} C e n t r e}$ for Animal Taxonomy and Ecology, Department of Zoology, Christ College, Irinjalakuda, Kerala 680125, India

*Corresponding author. Email: jomalamelcmi@gmail.com

Article history

Received: 25 August 2020; accepted 12 November 2020

Keywords:

Abundance; dominance; ecological guild; kleptoparasitic; spider

\begin{abstract}
A preliminary checklist of araneofauna of Pathiramanal Island is provided. A total of 147 species belonging to 26 families under 92 genera are documented. Tylorida ventralis (Thorell 1877) is considered to be the dominant species, and orb weavers are seen as the dominant guild. Araneidae, Salticidae, Theridiidae, Tetragnathidae and Thomisidae are five dominant families. The Shannon diversity, Simpson's (1-D) diversity, evenness and Chaol indices have been calculated. Seven species new to science such as Indopadilla insularis, Epeus triangulopalpis, Marengo sachintendulkar, Indomarengo chavarapater, Icius vikrambatrai, Piranthus planolancis (Salticidae) and Wolongia papafrancisi (Tetragnathidae) are documented as well as three genera and four species are added to the Indian spider taxonomy. The males of Meotipa picturara, Curubis tetrica and Pscellonus planus are described for the first time. Eight species are synonymized and redescribed. Mating plug formation in Argyrodes flavescens is reported for the first time. It is noted that spider species from Pathiramanl Island bear affinities with Oriental and Palearctic regions.
\end{abstract}

\section{INTRODUCTION}

A healthy ecosystem and its smooth functioning is an indicator of the potentiality of the biodiversity of that particular ecosystem (Pettersson 1996). Pathiramanal Island seems to be a healthy ecosystem with a tower of biodiversity of both plants and animals. As far as a local legend goes, Pathiramanal Island originated as a result of divine intervention. A young Brahmin (a person belonging to the Hindu higher caste worthy of priesthood) dived into Vembanad Lake to perform his evening prayers, and the water made way for land to rise from below, thus forming the enchanting Island of Pathiramanal which means 'sands of midnight'. A great charming look, wide waterfront, flourishing coconut palms, floating weeds and hyacinths, tiny birds building nests on weeds, and varieties of rare fauna and flora among the dense foliage of this tiny Island make it really a biodiversity hotspot. But, regrettably, no previous biodiversity studies have been done in Pathiramanal Island. An informal report indicates that the Island possesses some sort of fauna like odonates, birds, mammals, reptiles and arthropods (not a precise data). Arthropod diversity in this area remains undocumented, and a pilot study disclosed that it is a refuge for a rich volume of arachnids, to which spiders make a great contribution. But no organized work has been carried out to date on the taxonomy and diversity of spiders of this biologically unique ecosystem. 23 species of spiders have been named unofficially from this ecological zone. However, it is sure that a number of spiders have yet to be explored in this ecosystem, which prompts the need for an inventory of the spider fauna of this unique environmental sector. As spiders free us from most of insect pests like a key component to balancing the ecosystem both as prey and predator, they are very important animal taxa for humans (Benítez and Méndez 2011). In order to understand the potentiality of the Island and to conserve the spider species there, it is essential to explore the Island comprehensively. Therefore, this study is an attempt to throw light on the diversity, richness, abundance and dominance of spiders in Pathiramanal Island.

\section{MATERIALS AND METHODS}

\section{Study Area}

Pathiramanal Island (hereinafter, Island) is a small tropical island with an area of approximately $1 \mathrm{~km}^{2}$. It lies between the latitudes $9^{\circ} 37^{\prime} 07.11^{\prime \prime} \mathrm{N}$ and longitudes $76^{\circ} 23^{\prime} 04.95^{\prime \prime} \mathrm{E}$ (Figure 1). Though small in size, Pathiramanal Island is blessed with rich flora and fauna owing to the presence of a wide forest cover and thick vegetation (Figure 2). With respect to its geographical, climatic and ecological features, the Island harbours a rich amount of arachnids, of which spiders have a huge share. The temperature ranges from $28.6^{\circ} \mathrm{C}$ to $33.5^{\circ} \mathrm{C}$, with an annual mean of $31.0^{\circ} \mathrm{C}$ and plentiful rainfall in June-July (annual rainfall $>250 \mathrm{~mm}$ ). The dense vegeta- 
tion in the Island paves the way for strong winds which allows the circulation of good air thereby reducing the accumulation of pollutants. Till the late seventies of the 20th century, 14 worker families resided in the Island, who were later shifted to the mainland, and now the Island is non-inhabited. Recognizing the potential of the Island, Kerala Government took over the property in 1979 and later transferred it to the governance of the Tourism Department.

The diversity, richness and spatial distribution of spider species were investigated from October 2014 to September 2016. In the present study, four hours of sampling involved active searching for spiders, employing a combination of five collection methods such as aerial hand collection, ground hand collection, litter sampling, sweep netting and vegetation beating. All the specimens collected during the survey were transferred to a fixative ( $70 \%$ alcohol) for preservation. The sex and developmental stage of all trapped individuals were determined in the laboratory. Species-level identification was mainly made by looking at the genital features of the spiders. The palp and epigyne were dissected, and the epigyne was cleared in $10 \% \mathrm{KOH}$ for identifying the species. A detailed taxonomic study was carried out using the data provided by the World Spider Catalog (2020).
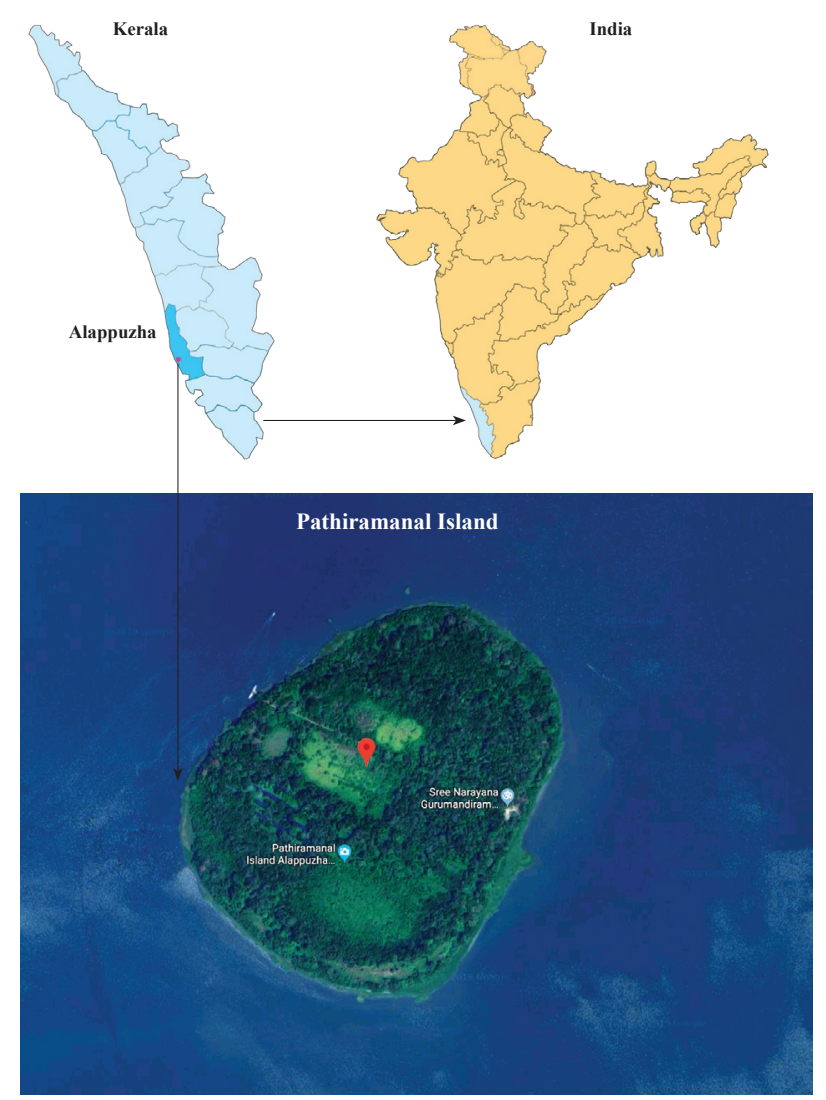

Figure 1. Map of the study area.

\section{Data analysis}

Statistical relevance of collected data was supported by calculating the following diversity indices:

Shannon-Weiner diversity index $\left(H^{\prime}\right)$ was calculated using the formula:

$H^{\prime}=-\sum\left[\left(p_{i}\right) \times \ln \left(p_{i}\right)\right]$,

where $-\sum=$ summation, $p_{i}=$ proportion of total sample represented by species $i$.

Simpson's diversity index $\left(D^{\prime}=1-D\right)$ was calculated using the formula:

$D=1-\sum n(n-1) / N(N-1)$

$D^{\prime}=1-D=1-\sum n(n-1)$

$N(N-\mathbf{1})$

where $-D=$ Simpson's index, $n=$ total number of organisms of a particular species, $N=$ total number of organisms of all species.

Evenness in species distribution was calculated using Simpson's formula:

$E=D / D_{\max }$,

where

$D=1 / \sum P_{i} 2$.

Chao1, an estimate of total species richness, was calculated using the formula:

Chaol $=S+F 1(F 1-1) /(2(\mathrm{~F} 2+1)$,

where $-F 1=$ the number of singleton species, $F 2=$ the number of doubleton species.

\section{RESULTS}

A total of 147 species of spiders belonging to 92 genera and 26 families were collected during the entire sampling period (Table 1). The Shannon diversity, Simpson's (1-D) diversity, evenness, and Chao1 indices were calculated to be $4.05,0.970,0.394$, and 149.3, respectively. The most dominant five families reported were Araneidae (22\%, 33 species), followed by Salticidae $(21.5 \%, 32$ species), Theridiidae (11\%, 16 species), Tetragnathidae $(9.5 \%, 14$ species) and Thomisidae $(5.5 \%, 8$ species $)$, while families such as Cheiracanthiidae, Gnaphosidae, Hersiliidae, Linyphiidae, Oonopidae, Philodromidae, Pholcidae, Pisauridae, Psechridae, Scytodidae, Sicariidae and Zodariidae were represented by only one species each. Tylorida ventralis was an abundant species throughout the study period. Out of 26 families, 23 are classified as entelegyne spiders and three (Oonopidae, Scytodidae, Sicariidae) are haplogyne spiders. Some families were more widely 

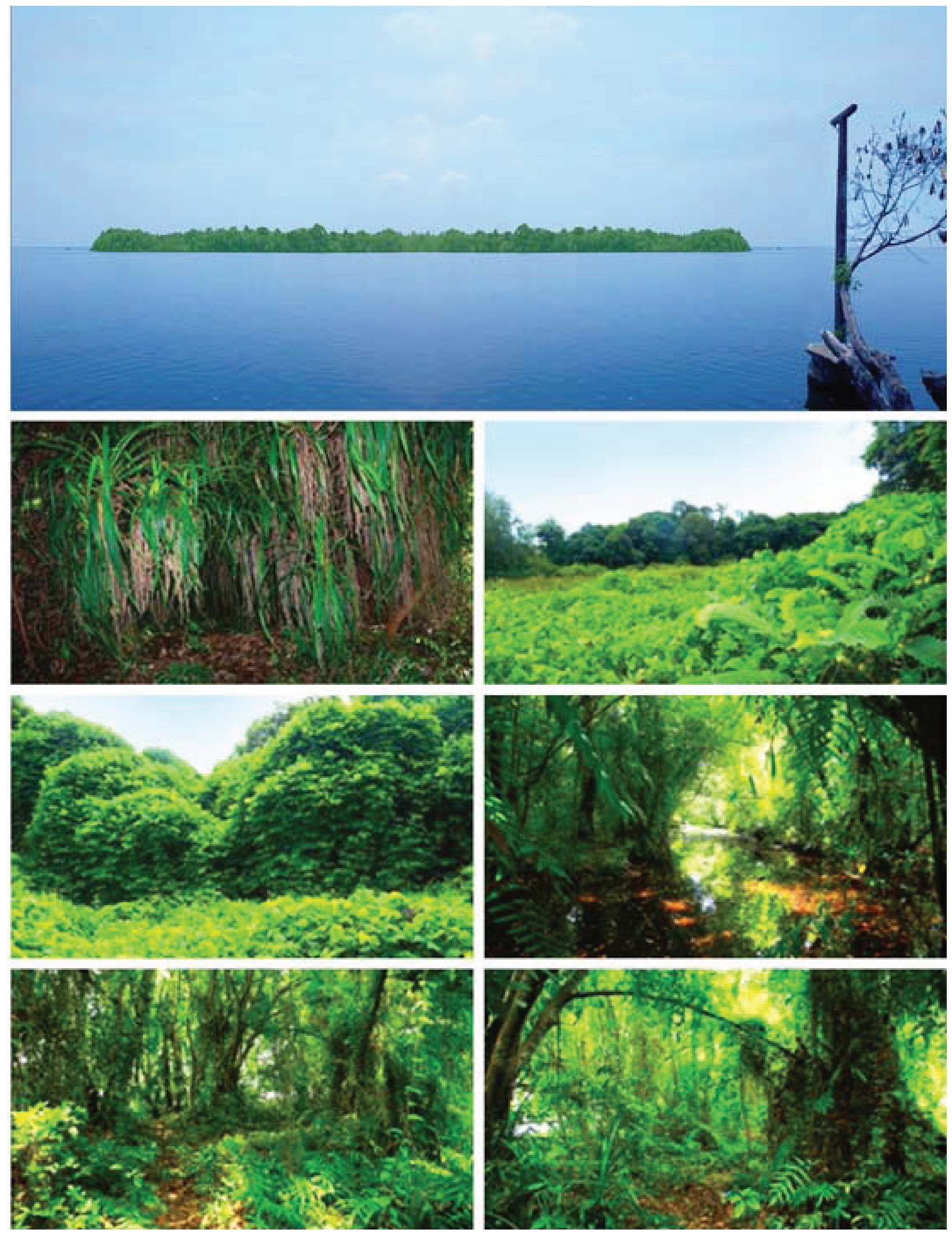

Figure 2. Pathiramanal Island and collection localities of the study area.

distributed throughout Pathiramanal Island, while others were restricted to one or a few habitat types. Two families were found in almost all sites: jumping spiders (Salticidae) and orb-web spiders (Araneidae). Two fami- lies were found in $75 \%$ of the sites: comb-footed spiders (Theridiidae) and long-jawed spiders (Tetragnathidae). Some other families found in more than $30 \%$ of all sites included wolf spiders (Lycosidae), crab spiders 
Table 1. Checklist of spiders of Pathiramanal island.

\begin{tabular}{|c|c|c|}
\hline Sl. No. & Family/Species & Remarks \\
\hline \multicolumn{3}{|c|}{ I. FAMILY ARANEIDAE Menge, 1890} \\
\hline 1 & Anepsion maritatum (O. Pickard-Cambridge, 1877) & New synonymy \\
\hline 2 & Araneus diadematus Clerck, 1757 & \\
\hline 3 & Araneus ellipticus (Tikader \& Bal, 1981) & \\
\hline 4 & Araneus sp.3 & \\
\hline 5 & Araneus sp.4 & \\
\hline 6 & Argiope aemula (Walckenaer, 1841) & \\
\hline 7 & Argiope anasuja Thorell, 1887 & \\
\hline 8 & Argiope pulchella Thorell, 1881 & \\
\hline 9 & Chorizopes anjanes Tikader, 1965 & \\
\hline 10 & Chorizopes sp.2 & \\
\hline 11 & Cyclosa bifida (Doleschall, 1859) & \\
\hline 12 & Cyclosa confraga (Thorell, 1892) & \\
\hline 13 & Cyclosa mulmeinensis (Thorell, 1887) & \\
\hline 14 & Cyclosa sp.4 & \\
\hline 15 & Cyrtophora cicatrosa (Stoliczka, 1869) & \\
\hline 16 & Cyrtophora citricola (Forsskal, 1775) & \\
\hline 17 & Cyrtophora moluccensis (Doleschall, 1857) & \\
\hline 18 & Cytrophora unicolor (Doleschall, 1857) & \\
\hline 19 & Eriovixia excelsa (Simon, 1889) & \\
\hline 20 & Eriovixia laglazei (Simon, 1877) & \\
\hline 21 & Eriovixia sp. 3 & \\
\hline 22 & Gasteracantha geminata (Fabricius, 1798) & Redescription \\
\hline 23 & Guizygiella nadleri (Heimer, 1984) & \\
\hline 24 & Herennia multipuncta (Doleschall, 1859) & \\
\hline 25 & Neoscona bengalensis (Tikader \& Bal, 1981) & \\
\hline 26 & Neoscona molemensis Tikader \& Bal, 1981 & \\
\hline 27 & Neoscona mukerjei Tikader, 1980 & \\
\hline 28 & Neoscona nautica (Koch, 1875) & \\
\hline 29 & Neoscona theisi (Walckenaer, 1841) & \\
\hline 30 & Neoscona vigilans (Blackwall, 1865) & \\
\hline 31 & Neoscona sp.7 & \\
\hline 32 & Parawixia dehaani (Doleschall, 1859) & First record \\
\hline 33 & Porcataraneus bengalensis (Tikader, 1975) & \\
\hline \multicolumn{3}{|c|}{ II. FAMILY CLUBIONIDAE Wagner, 1887} \\
\hline 34 & Clubiona drassodes O. Pickard-Cambridge, 1874 & \\
\hline 35 & Clubiona sp.2 & \\
\hline \multicolumn{3}{|c|}{ III. FAMILY CORINNIDAE Karsch, 1880} \\
\hline 36 & Castianeira zetes Simon, 1897 & \\
\hline 37 & Corinnoma severum (Thorell, 1877) & \\
\hline \multicolumn{3}{|c|}{ IV. FAMILY DICTYNIDAE Karsch, 1880} \\
\hline 38 & Dictyna sp. & \\
\hline 39 & Nigma sp. & \\
\hline \multicolumn{3}{|c|}{ V. FAMILY CHEIRACANTHIIDAE Lehtinen, 1967} \\
\hline 40 & Cheiracanthium danieli Tikader, 1975 & \\
\hline 41 & Cheiracanthium melanostomum (Thorell, 1895) & \\
\hline 42 & Cheiracanthium sp.3 & \\
\hline \multicolumn{3}{|c|}{ VI. FAMILY GNAPHOSIDAE Thorell, 1870} \\
\hline 43 & Urozelotus sp. & \\
\hline \multicolumn{3}{|c|}{ VII. FAMILY HERSILIIDAE Thorell, 1870} \\
\hline 44 & Hersilia savignyi Lucas, 1836 & \\
\hline \multicolumn{3}{|c|}{ VIII. FAMILY LINYPHIIDAE Blackwall, 1859} \\
\hline 45 & Nasoona crucifera (Thorell, 1895) & First record \\
\hline \multicolumn{3}{|c|}{ IX. FAMILY LIOCRANIDAE Simon, 1897} \\
\hline 46 & Oedignatha binoyii Reddy \& Patel, 1993 & \\
\hline 47 & Oedignatha scrobiculata Thorell, 1881 & \\
\hline
\end{tabular}




\begin{tabular}{|c|c|c|}
\hline Sl. No. & Family/Species & Remarks \\
\hline 48 & Hippasa greenalliae (Blackwall, 1867) & \\
\hline 49 & Lycosa sp. & \\
\hline 50 & Pardosa pseudoannulata (Bösenberg \& Strand, 1906) & \\
\hline 51 & Pardosa sumatrana (Thorell, 1890) & \\
\hline 52 & Pardosa sp. 3 & \\
\hline 53 & Zoica puellula (Simon, 1898) & \\
\hline \multicolumn{3}{|c|}{ XI. FAMILY OONOPIDAE Simon, 1890} \\
\hline 54 & Orchestina sp. & \\
\hline \multicolumn{3}{|c|}{ XII. FAMILY OXYOPIDAE Thorell, 1870} \\
\hline 55 & Hamataliwa sp. 1 & \\
\hline 56 & Hamataliwa sp. 2 & \\
\hline 57 & Oxyopes birmanicus Thorell, 1887 & \\
\hline 58 & Oxyopes javanus Thorell, 1887 & \\
\hline 59 & Oxyopes shweta Tikader, 1970 & \\
\hline 60 & Oxyopes sunandae Tikader, 1970 & \\
\hline 61 & Oxyopes sp.5 & \\
\hline \multicolumn{3}{|c|}{ XIII. FAMILY PHILODROMIDAE Thorell, 1870} \\
\hline 62 & Psellonus planus Simon, 1897 & Redescription \\
\hline \multicolumn{3}{|c|}{ XIV. FAMILY PHOLCIDAE C. L. Koch, 1850} \\
\hline 63 & Crossopriza lyoni (Blackwall, 1867) & \\
\hline 64 & Pholcus sp. & \\
\hline \multicolumn{3}{|c|}{ XV. FAMILY PISAURIDAE, Simon, 1890} \\
\hline 65 & Dendrolycosa gitae Tikader, 1970 & \\
\hline \multicolumn{3}{|c|}{ XVI. FAMILY PSECHRIDAE, Simon, 1890} \\
\hline 66 & Fecenia protensa Thorell, 1891 & New Synonymy \\
\hline \multicolumn{3}{|c|}{ XVII. FAMILY SALTICIDAE, Blackwall, 1841} \\
\hline 67 & Brettus cingulatus Thorell, 1895 & \\
\hline 68 & Carrhotus sannio (Thorell, 1877) & \\
\hline 69 & Carrhotus viduus (C. L. Koch, 1846) & \\
\hline 70 & Chalcotropis pennata Simon, 1902 & \\
\hline 71 & Cosmophasis sp. & \\
\hline 72 & Curubis tetrica Simon, 1902 & Redescription \\
\hline 73 & Epeus tener (Simon, 1877 ) & \\
\hline 74 & Epeus triangulopalpis Malamel et al. .2018 & New species \\
\hline 75 & Epeus sp.3 & \\
\hline 76 & Epocilla aurantiaca (Simon, 1885) & \\
\hline 77 & Evarcha pococki Zabka, 1985 & First record \\
\hline 78 & Hyllus semicupreus (Simon, 1885) & \\
\hline 79 & Icius vikrambatrai Malamel et al. 2018 & New species \\
\hline 80 & Indomarengo chavarapater Malamel et al. 2018 & New species \\
\hline 81 & Indopadilla insularis Malamel et al. 2015 & New species \\
\hline 82 & Marengo sachintendulkar Malamel et al. 2018 & New species \\
\hline 83 & Myrmaplata plataleoides (O. Pickard-Cambridge, 1869) & \\
\hline 84 & Myrmarachne melanocephala MacLeay, 1839 & \\
\hline 85 & Myrmarachne robusta (Peckham \& Peckham, 1892) & \\
\hline 86 & Myrmarachne prava Tikader,1973 & \\
\hline 87 & Phaeacius lancearius (Thorell, 1895) & \\
\hline 88 & Phintella vittata (C. L. Koch, 1846) & \\
\hline 89 & Piranthus planolancis Malamel et al. 2018 & New species \\
\hline 90 & Plexippus paykulli (Audouin, 1826) & \\
\hline 91 & Portia fimbriata (Doleschall, 1859) & \\
\hline 92 & Rhene danieli Tikader, 1973 & \\
\hline 93 & Rhene flavicomans Simon, 1902 & \\
\hline 94 & Rhene flavigera (Koch, 1846) & \\
\hline 95 & Siler semiglaucus Simon, 1961 & \\
\hline 96 & Telamonia dimidiata (Simon, 1899) & \\
\hline 97 & Thiania bhamoensis Thorell, 1887 & \\
\hline 98 & Uroballus sp. & \\
\hline
\end{tabular}




\begin{tabular}{|c|c|c|}
\hline Sl. No. & Family/Species & Remarks \\
\hline \multicolumn{3}{|c|}{ XVIII. FAMILY SCYTODIDAE, Blackwall, 1864} \\
\hline 99 & Scytodes thoracica (Latreille, 1802) & \\
\hline \multicolumn{3}{|c|}{ XIX. FAMILY SICARIIDAE Keyserling, 1880} \\
\hline 100 & Loxosceles rufescens (Dufour, 1820) & \\
\hline \multicolumn{3}{|c|}{ XX. FAMILY SPARASSIDAE Berkatu, 1891} \\
\hline 101 & Heteropoda venatoria (Linnaeus, 1767) & \\
\hline 102 & Olios sp.1 & \\
\hline 103 & Olios sp.2 & \\
\hline 104 & Thelcticopis virescens Pocock, 1901 & \\
\hline \multicolumn{3}{|c|}{ XXI. FAMILY TETRAGNATHIDAE Menge, 1866} \\
\hline 105 & Dolichognatha sp. & \\
\hline 106 & Leucauge decorata (Blackwall, 1864) & \\
\hline 107 & Leucauge granulata (Walckenaer, 1841) & New synonymy \\
\hline 108 & Tetragnatha cochinensis Gravely, 1921 & \\
\hline 109 & Tetragnatha javana (Thorell, 1890) & \\
\hline 110 & Tetragnatha mandibulata Walckenaer, 1841 & \\
\hline 111 & Tetragnatha versicolor Walckenaer, 1841 & \\
\hline 112 & Tetragnatha viridorufa Gravely, 1921 & \\
\hline 113 & Tetragnatha sp.6 & \\
\hline 114 & Tetragnatha sp.7 & \\
\hline 115 & Tetragnatha sp.8 & \\
\hline 116 & Tylorida striata (Thorell, 1877) & \\
\hline 117 & Tylorida ventralis (Thorell, 1877) & \\
\hline 118 & Wolongia papafrancisi Malamel et al. 2018 & New species \\
\hline \multicolumn{3}{|c|}{ XXII. FAMILY THERIDIIDAE Sundevall, 1833} \\
\hline 119 & Achaearanea sp. & \\
\hline 120 & Argyrodes argentatus O. Pickard-Cambridge, 1880 & \\
\hline 121 & Argyrodes bonadea (Karsch, 1881) & \\
\hline 122 & Argyrodes flavescens O. Pickard-Cambridge, 1880 & \\
\hline 123 & Argyrodes gazedes Tikader, 1970 & \\
\hline 124 & Ariamnes flagellum (Doleschall, 1857) & \\
\hline 125 & Chrysso sp. & \\
\hline 126 & Coleosoma blandum O. Pickard-Cambridge, 1882 & \\
\hline 127 & Euryopis sp. & \\
\hline 128 & Meotipa picturata Simon, 1895 & Redescription \\
\hline 129 & Nihonhimea mundula (L. Koch, 1872) & \\
\hline 130 & Parasteatoda tepidariorum (C.L Koch, 1872) & \\
\hline 131 & Parasteatoda $\mathrm{sp} .2$ & \\
\hline 132 & Steatoda $\mathrm{sp}$ & \\
\hline 133 & Rhomphaea sp. & \\
\hline 134 & Theridion manjithar Tikader, 1970 & \\
\hline \multicolumn{3}{|c|}{ XXIII. FAMILY THOMISIDAE Sundevall, 1833} \\
\hline 135 & Amyciaea forticeps (Cambridgae, 1873) & \\
\hline 136 & Epidius parvati Benjamin, 2000 & First record \\
\hline 137 & Epidius sp.2 & \\
\hline 138 & Indoxysticus minutus (Tikader, 1960) & \\
\hline 139 & Misumenops sp. & \\
\hline 140 & Thomisus lobosus Tikader, 1965 & \\
\hline 141 & Thomisus projectus Tikader, 1960 & \\
\hline 142 & Thomisus sp.3 & \\
\hline \multicolumn{3}{|c|}{ XXIV. FAMILY TRACHELIDAE Simon, 1897} \\
\hline 143 & Trachelas sp. & \\
\hline 144 & Utivarachna sp. & \\
\hline \multicolumn{3}{|c|}{ XXV. FAMILY ULOBORIDAE, Thorell, 1869} \\
\hline 145 & Uloborus krishnae Tikader, 1970 & \\
\hline 146 & Zosis geniculata (Olivier, 1789) & \\
\hline \multicolumn{3}{|c|}{ XXVI. FAMILY ZODARIIDAE Thorell, 1881} \\
\hline 147 & Tropizodium virudorbium Prajapati et al. 2016 & \\
\hline
\end{tabular}


(Thomisidae), huntsman spiders (Sparassidae), and families that were only found in a single site included flat-bellied ground spiders (Gnaphosidae), running crab spiders (Philodromidae), violin spiders (Sicariidae) and mesh-web spiders (Dictynidae).

The spiders of Pathiramanal Island can be divided into seven feeding guilds based on the foraging behaviour. They are ambushers, foliage runners, ground runners, orb weavers, sheet web builders, space web builders and stalkers. The dominant guild was of orb weavers (33\%), followed by stalkers (27\%), space web builders (13\%), ambushers (11\%), ground runners (9\%), foliage runners $(6 \%)$ and sheet web builders (1\%) (Table 2, Figure 3).

One of the most notable achievements of the study is the discovery and documentation of seven species new to science, such as Indopadilla insularis, Epeus triangulopalpis, Marengo sachintendulkar, Indomarengo chavarapater, Icius vikrambatrai, Piranthus planolancis (Salticidae) and Wolongia papafrancisi (Tetragnathidae) (Figure 4, Table 3). Three genera and four species are documented as new records from Indian region (Figure 5) and 124 species are newly recorded from Pathiramanal Island. It was surprising to note the presence of the poisonous spider Loxosceles rufescens coming under the family Sicariidae.

Mating plug formation is often common in the genus
Argyrodes since these spiders are found both sexes together and live as kleptoparasites in the webs of large orb-weaving spiders (Koh and Li 2002). Although a number of reports on mating plug formation in this genus had been done before, mating plug formation in the sixth species of Argyrodes (A. flavescens) was observed for the first time during this study based on the specimens collected from Pathiramanal Island (Figure 6).
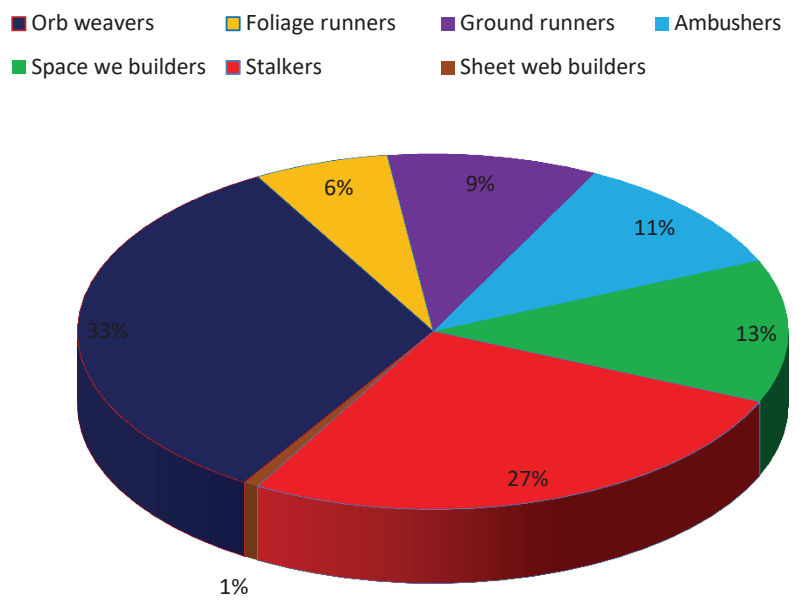

Figure 3. Guild structure of spiders.

Table 2. Total number of families, genera, species and functional guilds of spiders in Pathirmanal island.

\begin{tabular}{|c|c|c|c|c|}
\hline Sl. No. & Family & No. of genera & No. of species & Guild \\
\hline \multicolumn{5}{|c|}{ Suborder Araneomorphae } \\
\hline 1 & FAMILY ARANEIDAE Menge, 1890 & 13 & 33 & Orb weavers \\
\hline 2 & FAMILY CLUBIONIDAE Wagner, 1887 & 1 & 2 & Foliage runners \\
\hline 3 & FAMILY CORINNIDAE Karsch, 1880 & 2 & 2 & Ground runners \\
\hline 4 & FAMILY DICTYNIDAE Karsch, 1880 & 2 & 2 & Foliage runners \\
\hline 5 & FAMILY CHEIRACANTHIIDAE Wagner, 1887 & 1 & 3 & Foliage runners \\
\hline 6 & FAMILY GNAPHOSIDAE Thorell, 1870 & 1 & 1 & Ground runners \\
\hline 7 & FAMILY HERSILIIDAE Thorell, 1870 & 1 & 1 & Ambushers \\
\hline 8 & FAMILY LINYPHIIDAE Blackwall, 1859 & 1 & 1 & Space web builders \\
\hline 9 & FAMILY LIOCRANIDAE Simon, 1897 & 1 & 2 & Ground runners \\
\hline 10 & FAMILY LYCOSIDAE Sundevall, 1833 & 4 & 6 & Ground runners \\
\hline 11 & FAMILY OONOPIDAE Simon, 1890 & 1 & 1 & Ground runners \\
\hline 12 & FAMILY OXYOPIDAE Thorell, 1870 & 2 & 7 & Stalkers \\
\hline 13 & FAMILY PHILODROMIDAE Thorell, 1870 & 1 & 1 & Ambushers \\
\hline 14 & FAMILY PHOLCIDAEC. L. Koch, 1850 & 2 & 2 & Space web builders \\
\hline 15 & FAMILY PISAURIDAE, Simon, 1890 & 1 & 1 & Ambushers \\
\hline 16 & FAMILY PSECHRIDAE, Simon, 1890 & 1 & 1 & Sheet web builders \\
\hline 17 & FAMILY SALTICIDAE, Blackwall, 1841 & 25 & 32 & Stalkers \\
\hline 18 & FAMILY SCYTODIDAE, Blackwall, 1864 & 1 & 1 & Ground runners \\
\hline 19 & FAMILY SICARIIDAE Keyserling, 1880 & 1 & 1 & Ambushers \\
\hline 20 & FAMILY SPARASSIDAE Berkatu, 1891 & 3 & 4 & Ambushers \\
\hline 21 & FAMILY TETRAGNATHIDAE Menge, 1866 & 5 & 14 & Orb weavers \\
\hline 22 & FAMILY THERIDIIDAE Sundevall, 1833 & 12 & 16 & Space web builders \\
\hline 23 & FAMILY THOMISIDAE Sundevall, 1833 & 5 & 8 & Ambushers \\
\hline 24 & FAMILY TRACHELIDAE Simon, 1897 & 2 & 2 & Foliage runners \\
\hline 25 & FAMILY ULOBORIDAE, Thorell, 1869 & 2 & 2 & Orb weavers \\
\hline 26 & FAMILY ZODARIIDAE Thorell, 1881 & 1 & 1 & Ground runners \\
\hline \multicolumn{2}{|l|}{ Total } & 92 & 147 & \\
\hline
\end{tabular}



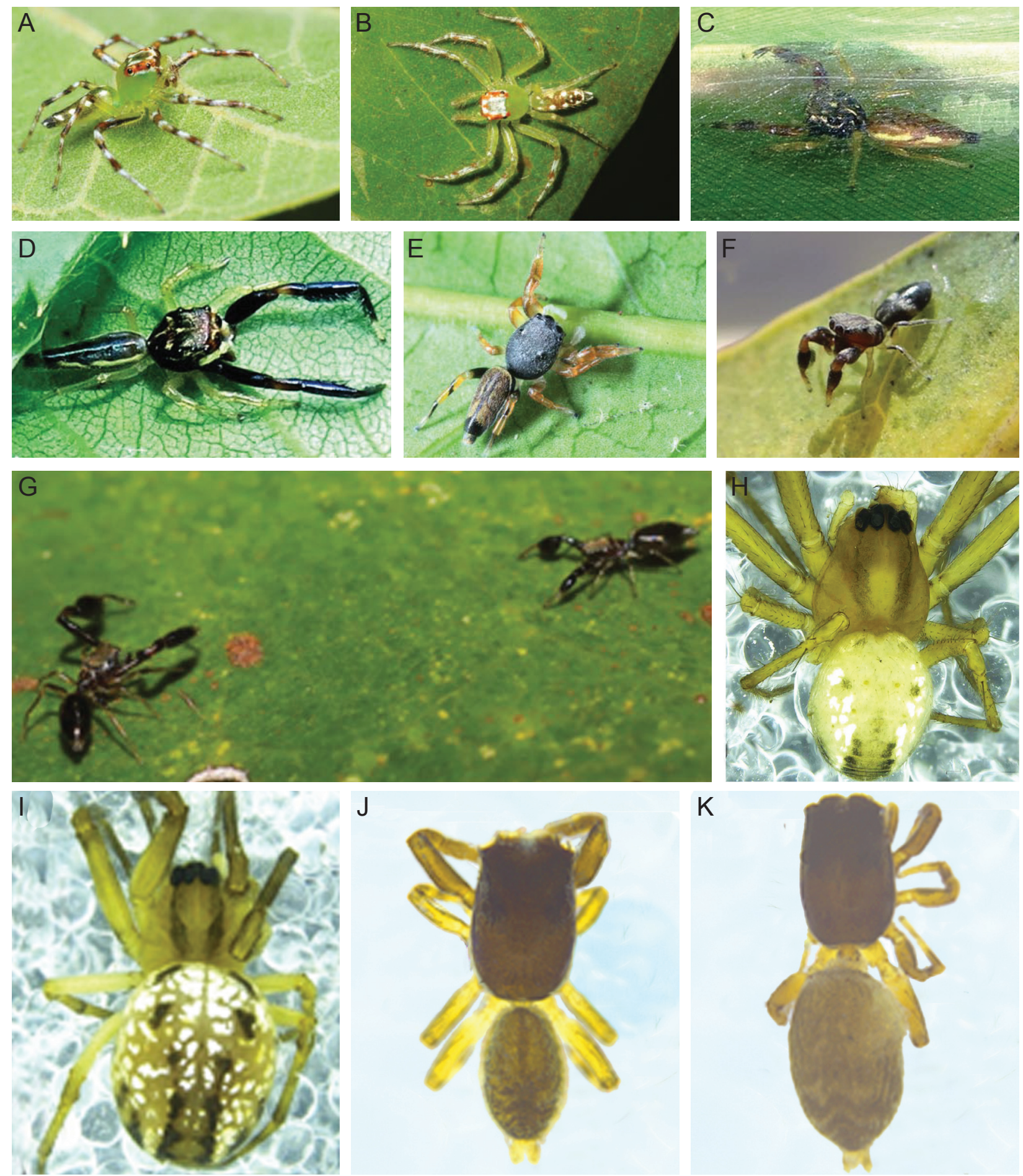

\begin{abstract}
J
\end{abstract}

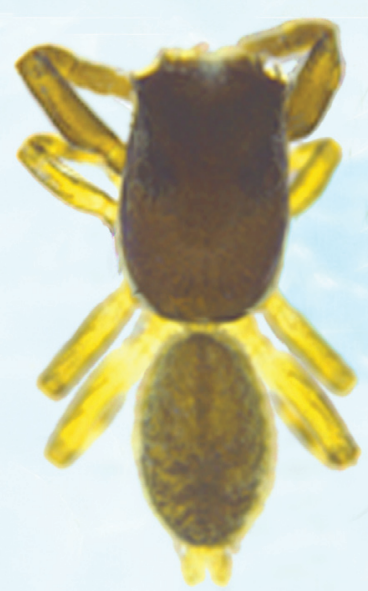

$\mathrm{K}$

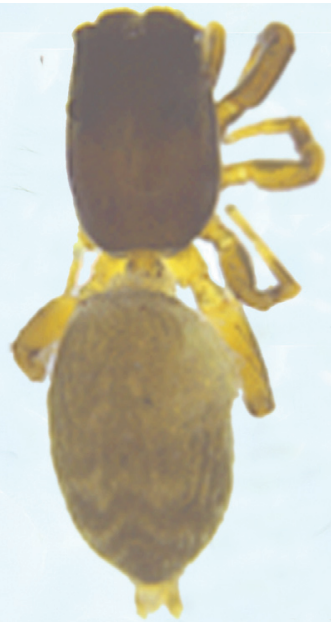

Figure 4. New species described (field photos): A, B, male and female of Epeus triangulopalpis; C, D, male and female of Indopadilla insularis; E, female of Piranthus planolancis; F, female of Marengo sachintendulkar; G, male and female of Indomarengo chavarapater, H, I, male and female of Wolongia papafrancisi; J, K, male and female of Icius vikrambatrai.

\section{DISCUSSION}

The present study is the first comprehensive documentation of the spider fauna in Pathiramanal Island. The present study revealed that the total spider diversity of Pathiramanal Island represents $10 \%$ of total species, $22 \%$ of total genus and $43 \%$ of total family diversity reported from India (Sebastian and Peter 2009) and accounts for $35 \%$ of total species, $40 \%$ of total genus and $60.5 \%$ of total family diversity from Kerala (Jobi and Sebastian, in preparation). However, it seems that a good number of species remains that probably are new species but cannot be described due to lack of proper literature on these taxa. A high number of species recorded indicates a rich spider diversity of this region which was also indicated by Shannon diversity 

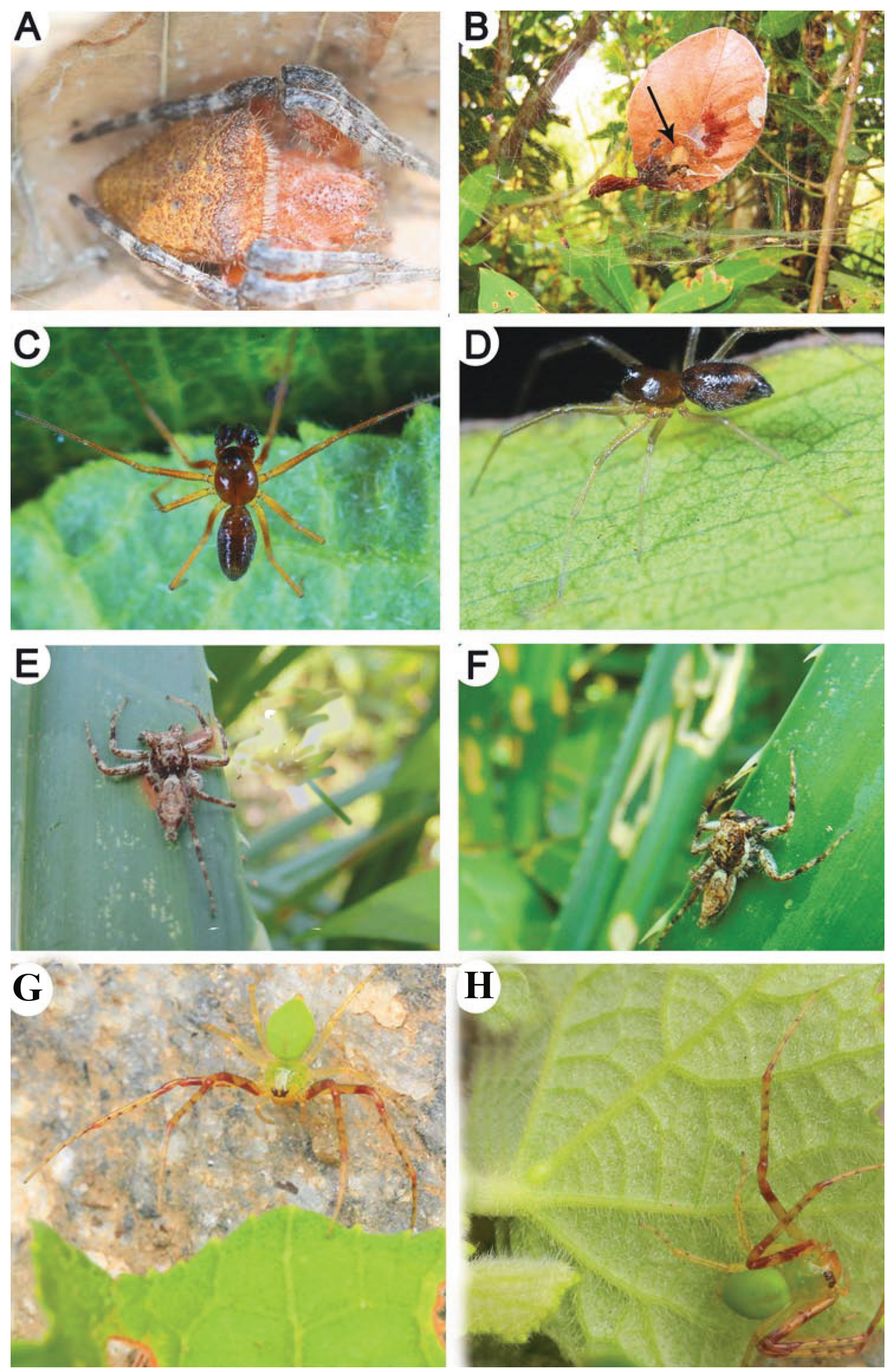

Figure 5. New records from India: A, B, female of Cytrophora unicolor; C, D, male and female of Nasoona crucifera; E, F, male of Phaeacius lancearius; G, H, female of Epidius parvati. 
Table 3. New species described.

\begin{tabular}{|c|c|l|l|c|}
\hline No. & \multicolumn{1}{|c|}{ Family } & \multicolumn{1}{|c|}{ Genus } & \multicolumn{1}{|c|}{ Species } & Status \\
\hline 1 & Salticidae & Epeus & triangulopalpis & $\begin{array}{c}\text { New } \\
\text { species }\end{array}$ \\
\hline 2 & $\#$ & Icius & vikrambatrai &, \\
\hline 3 & $\#$ & Indomarengo & chavarapater &, \\
\hline 4 &, & Indopadilla & insularis &, \\
\hline 5 &, & Marengo & sachintendulkar &, \\
\hline 6 & $\#$ & Piranthus & planolancis &, \\
\hline 7 & Tetragnathidae & Wolongia & papafrancisi &, \\
\hline
\end{tabular}
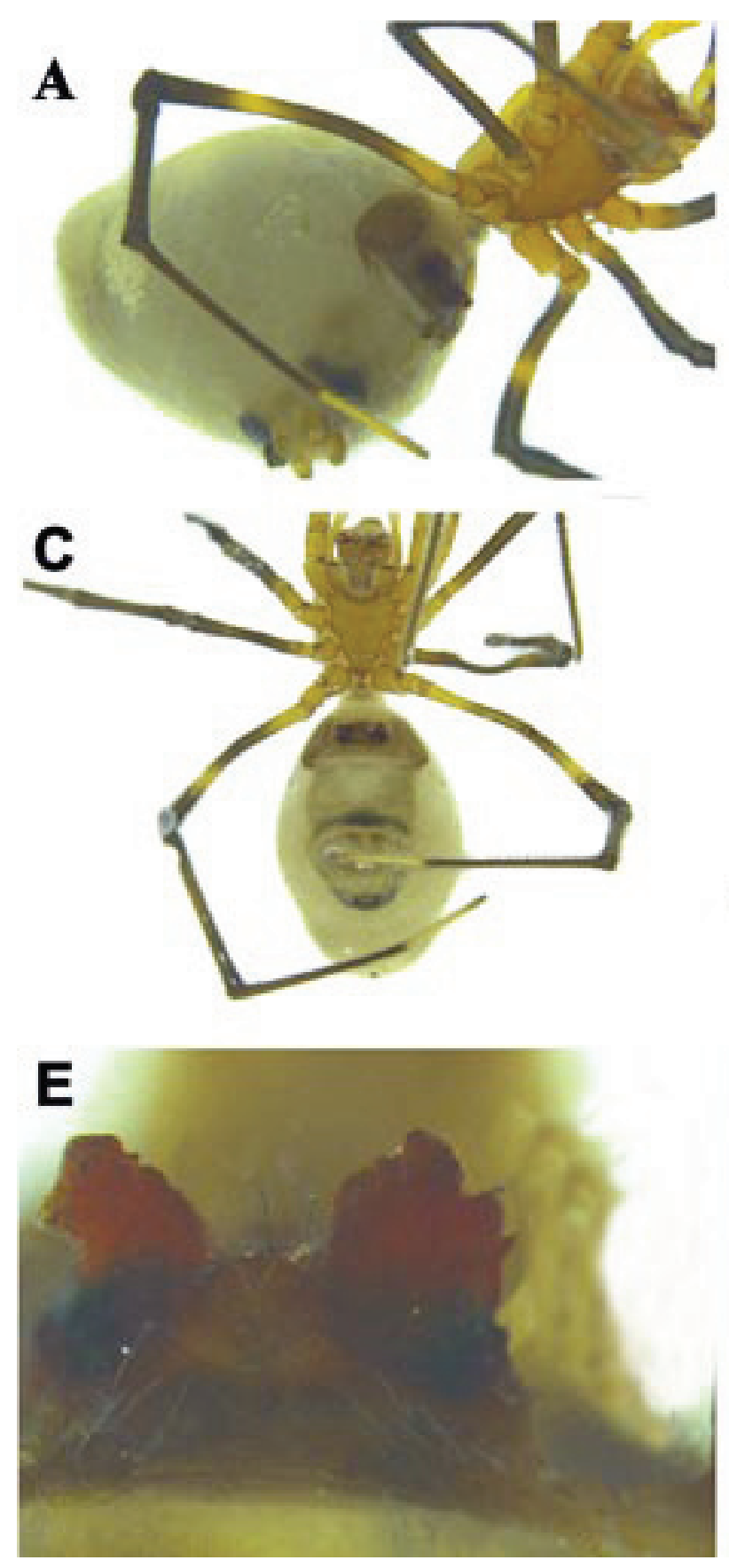

Figure 6. A-D, mating plug formation in Argyrodes flavescens; E, epigyne with mating plug (dorsal view); F, epigyne without mating plug.

index $(\mathrm{H}=4.05)$ and Simpson's diversity index $(1-\mathrm{D}=$ $0.970)$. Even though the observed species richness was 147, the estimated species richness (Chao1) was found to be 149.3 , which designates that a number of species could be collected from a thorough sampling of a long period. The existence of spiders is mainly dependent on the habitat they live in and select based on the physiological factors of the habitat, availability of prey and the apt site for web building.

The guild study was done to form the basis of community organization of spiders in Pathiramanal Island, and the study yielded seven ecological guilds based

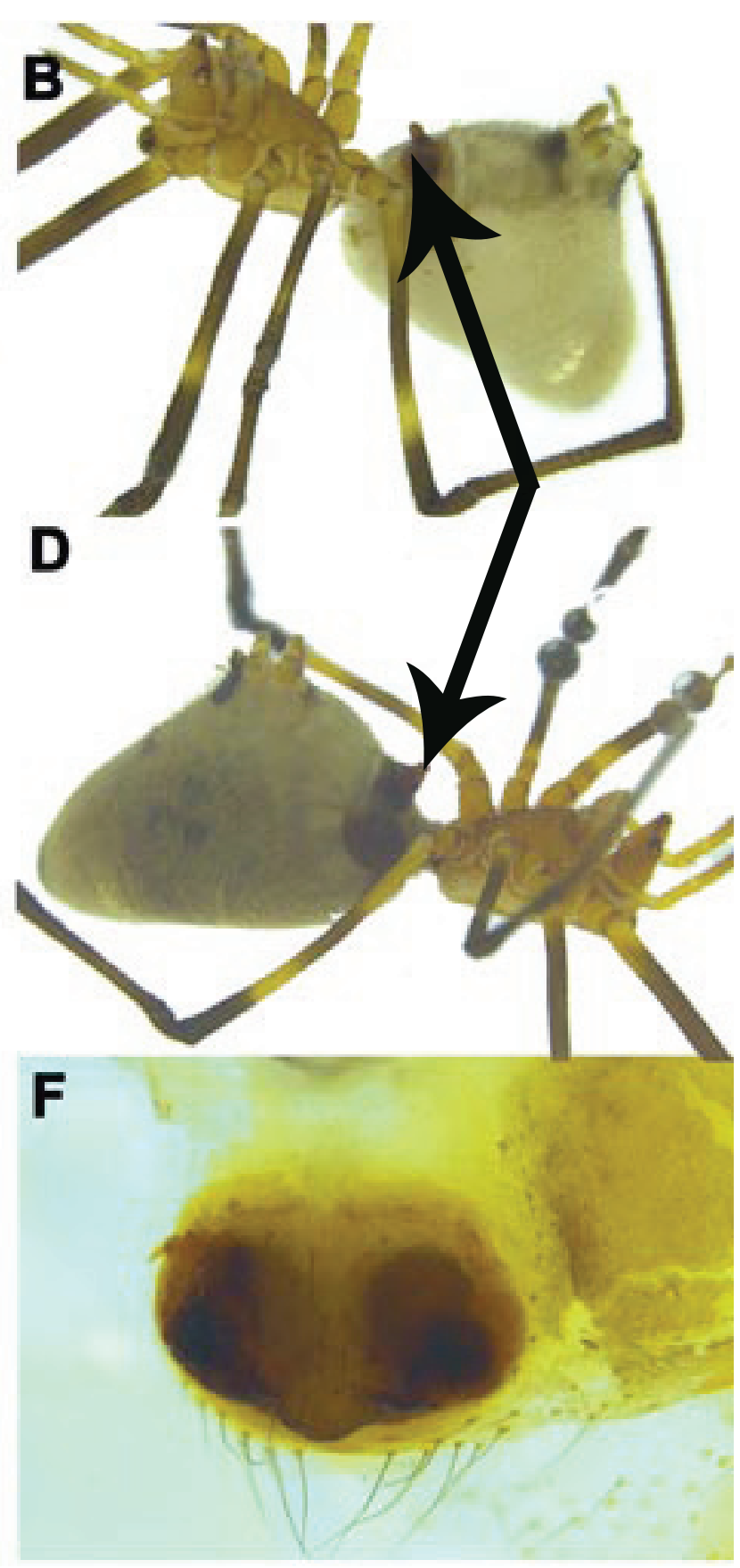


on their foraging mode (Figure 3). Orb weavers were the dominant guild (33\%) including the families such as Araneidae, Tetragnathidae and Uloboridae, and this might be due to the collecting localities of Pathiramanal Island that take care of shelter, reproductive behaviour and foraging of these orb webs. The sheet web builders were the lowest guild type (1\%) with the family Psechridae. The presence of the poisonous spider Loxosceles rufescens in the Island reminds that tourists should be aware of these spiders because of their ability to cause skin necrosis or loxoscelism with their necrotizing venom.

As Argyrodes flavescens is a social spider and often seen with both sexes together, it is easier for male spiders to make plugs in female openings. This observation suggests that their nature of living is critical in plug formation. The reason behind mating plug formation being common in this species could be its kleptoparasitic nature in the webs of host species and the usual presence of males.

According to Holloway (1974), Indian fauna formed as a result of displacement by invaders from other regions of the Oriental region after its separation from Gondwanaland and merger with Asia. So spider species from Pahiramanal Island also bear affinities with Oriental and Palearctic regions since it is a part of Vembanad Lake, a Ramsar site in India. Some species of the genera $\mathrm{Ar}$ giope, Cyclosa, Eriovixia, Gasteracantha (family Araneidae); Tylorida, Tetragnatha (family Tetragnathidae) exhibit Sri Lankan affinities. Some species of genera Argiope, Cyclosa, Eriovixia, Gasteracantha, Neoscona (family Araneidae); Leucauge, Tetragnatha (family Tetragnathidae) bear Oriental affinities. An araneid species Eriovixia laglaizei shows Palearctic affinities.

Spiders can easily colonize any habitat, especially the isolated lands like Pathiramanal Island, as they are able to disperse over long distances using wind currents ("ballooning') in general. The difficulty in spider biodiversity assessment in Pathiramanal Island seems to be related to the lack of historical expeditions focusing on this group of arthropods. High richness and variability as well as the rate of new species records and new species descriptions indicate a satisfactory picture of spider diversity in Pathiramanal Island.

Pathiramanal Island is a land of a dense forest cover and thick vegetation. This investigation showed that an important determinant of spider species richness and diversity in the Island is habitat diversity. The data revealed that structurally vegetation which is more diverse supports a higher number of spider species, which could be explained by a greater variety of available niches within more complex vegetation. Overall, the results showed that vegetation differed from one Island spot to another, and spider composition was also highly dissimilar in dif- ferent plots of the Island. This study report emphasizes the importance of maintaining a mosaic-like pattern in the habitat, because different vegetation patches (e.g. thick/thin vegetation, tall/short vegetation) can provide habitats for very different spider assemblages and thus enhance the overall spider diversity.

\section{Faunal affinities of spiders collected from Pathira- manal Island}

The araneofauna of Pathiramanal Island bears affinities with Oriental and Palearctic regions and with the fauna of Sri Lanka. It is hypothesized that Indian biota is the result of displacement by invaders from other regions of the Oriental region after its separation from Gondwanaland and merger with Asia (Holloway 1974). Species such as Araneus ellipticus, Argiope aemula, A. anasuja, A. pulchella, Cyclosa bifida, C. confraga, Cyrtophora cicatrosa, C. moluccensis, Eriovixia laglaizei, Gasteracantha geminata, Herennia multipuncta, Neoscona molemensis, $N$. vigilans, $N$. nautica, Parawixia dehanii (family Araneidae); Clubiona drassodes (family Clubionidae); Castianeira zetes (family Corinnidae); Stegodyphus sarasinorum (family Eresidae); Hippasa greenalliae, Pardosa pseudoannulata, P. sumatrana (family Lycosidae); Cheiracanthium melanostomum (family Cheiracanthiidae); Oxyopes birmanicus, O. javanus (family Oxyopidae); Fecenia protensa (family Psechridae); Carrhotus viduus, Menemerus bivittatus, Myrmarachne melanocephala, Phintella vittata, Plexippus paykulli, Portia fimbriata, Rhene flavigera, Siler semiglaucus, Telamonia dimdiata, Thiania bhamoensis (family Salticidae); Heteropoda venatoria (family Sparassidae); Argyrodes flavescens, Ariamnes flagellum, Nihonhimea mundula (family Theridiidae) and Zosis geniculata (family Uloboridae) bear Oriental affinities.

Palearctic affinities are shown by species like Eriovixia laglaizei, Porcataraneus bengalensis (family Araneidae); Clubiona drassodes (family Clubionidae); Hippasa greenalliae (family Lycosidae); Oxyopes birmanicus, O. Shweta (family Oxyopidae); Myrmaplata plataleoides (family Salticidae); Leucauge decorata, Tetragnatha javana (family Tetragnathidae) and Argyrodes flavescens (family Theridiidae).

It was studied by Bossuyt et al. (2004) analyzing the links between the Western Ghats (southern India) and Sri Lanka, using multiple vertebrate and invertebrate groups, that Sri Lankan fauna derived from mainland India. The current study partially approves this hypothesis from the remarkable similarities exhibited between the spider fauna of Pathiramanal and Sri Lanka. Species such as Argiope aemula, A. anasuja, A. pulchella, Cyclosa confraga, Cyrtophora cicatrosa, C. moluccensis, Eriovixia laglaizei, Gasteracantha geminata, Herennia multipuncta, Parawixia dehanii (family Araneidae); 
Stegodyphus sarasinorum (family Eresidae); Hersilia savigyni (family Hersiliidae); Hippasa greenalliae (family Lycosidae); Hylus semicupreus, Myrmaplata plataleoides (family Salticidae); Heteropoda venatoria (family Sparassidae); Argyrodes flavescens, Ariamnes flagellum, Nihonhimea mundula (family Theridiidae) bear affinities to the Island fauna of Sri Lanka.

\section{CONCLUSION}

Until now, Pathiramanal Island was considered to be home to only 23 spider species due to the lack of studies in this region. However, the current survey of the araneofauna in this remote Island has challenged this view. A high species diversity of spiders in Pathiramanal Island can be attributed to a high floral diversity of the Island, which sustains high faunal diversity by providing diverse microhabitat, especially for invertebrates. Contrasting to other ecologically important zones, there is no previous work to compare the spider diversity. This indicates the need for a further systematic sampling in this area. The presence of diverse habitats like big trees, bushes and grasslands in this ecosystem is a revelation to show Pathiramanal Island harbours many smaller but diverse environmental niches, which makes the Island an important centre of speciation in the Vembanad, a Ramsar site. Since Pathiramanal Island shows a surprisingly diverse spider community, further research should be encouraged in this biome to maintain and manage this high diversity, and the factors other than habitat type, which are important in influencing diversity, need to be investigated.

\section{ACKNOWLEDGEMENTS}

The authors are grateful to Fr. Prasanth Palackappillil CMI, Principal, Sacred Heart College, Thevara, Cochin, for providing all facilities for completing this work. Many thanks to Atul Vartak, Naffin K. S. and Jimmy Paul for the field photographs. Millions of thanks to the anonymous reviewers for their constructive comments to develop this work. The first author greatly acknowledges the IDEA WILD team for the financial assistance to proceed the work.

\section{REFERENCES}

Benítez, S. P. and M. Méndez. 2011. Effects of land management on the abundance and richness of spiders (Araneae): A meta-analysis. Biological Conservation 144 (2): 683-691.

Bossuyt, F., M. Meegaskumbura, N. Beenaerts, D. J. Gower, R. K. Pethiyagoda, A. Mannaert, M. Wilkinson, M. M. Bahir, K. Manamendra-Arachchi, P. K. L. Roelants, C. J. Ng, Schneider, O. V. Oommen, and M. C. Milinkovitch. 2004. Local endemism within the Western Ghats-Sri Lanka biodiversity hotspot. Science 306: 479-481.

Holloway, J. D. 1974. Chapter 15. The biogeography of the Indian butterflies. In Ecology and Biogeography in India, edited by Mani M., 473-499. The Hauge: W. Junk Publishers.

Koh, T., and D. Li. 2002. Population characteristics of a kleptoparasitic spider Argyrodes flavescens (Araenae: Theridiidae) and its impact on a host spider Nephila pilipes (Araneae: Tetragnathidae) from Singapore. The Raffles Bulletin of Zoology 50: 153-160.

Pettersson, R. B. 1996. Effect of forestry on the abundance and diversity of arboreal spiders in the boreal spruce forest. Ecography 19 (3): 221-228.

Sebastian, P. A., and K. V. Peter. 2009. Spiders of India. Hyderabad: Universities press. 614 pp.

World Spider Catalog. 2020. World Spider Catalog. Version 21.0. Natural History Museum Bern, online at http:// wsc.nmbe.ch, accessed on \{accessed on 31 March\}. 